\title{
Behavioural Non-Motor Fluctuations in Parkinson Disease Why it is important for psychiatrists to recognize them
}

\author{
Alberto Marques¹, David Teixeira1, Bruna Melo1, Carla Alves Pereira1, Lúcia Costa11, João Brás', Rui \\ Sousa', Sofia Ribeiro Pereira1
}

1 Department of Psychiatry and Mental Health, Centro Hospitalar Tondela-Viseu, EPE., Viseu, Portugal

OBJECTIVES: To present a clinical case of behavioural nonmotor fluctuations in Parkinson Disease (PD) and their difficult recognition in daily practise.

BACKGROUND AND AIMS: PD fluctuations (or "off" states) are related to the wearing-off phenomenon of levodopa, correlating with its falling plasma concentration. The term generally refers to the gradual return of parkinsonism, although sensory/autonomic/behavioural ( $\mathrm{SAB}$ ) fluctuations can present as an "off" without any motoric parkinsonic signs. SAB "offs" often go unrecognized and are extremely poorly tolerated. They consist of any combination of: pain, akathisia, drenching sweating, abdominal bloating, urinary urgency, dyspnea, dysphoria, depression, anxiety and panic. Smoothing out levodopa's plasma concentration will relieve $S A B$ fluctuations and prevent new episodes.
MATERIALS AND METHODS: Patient's clinical files consultation and scientific bibliography search using the keywords Parkinson Disease, Non-motor Symptoms, Levodopa Fluctuations.

RESULTS: A 72 year-old man, with PD and no previous psychiatric history, resorted to the emergency service due to suicidal ideation that began in the same day. The accompanying complaints of agitation, shortness of breath, anguish and sadness were too difficult to tolerate. After sedation with partial relief, he was referred to a psychiatry appointment. A few months later he experienced a new crisis. This time sedation was ineffective and the symptoms only remitted after the administration of levodopa.

\section{Long-Term Complications of Levodopa Treatment}

\section{Fluctuations ("off" states)}

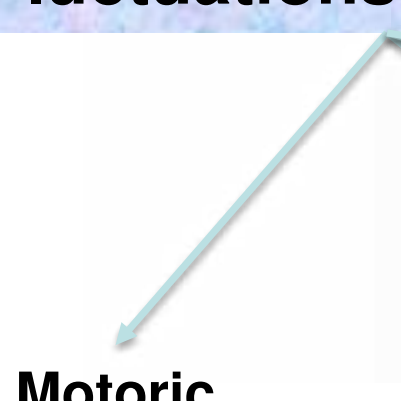

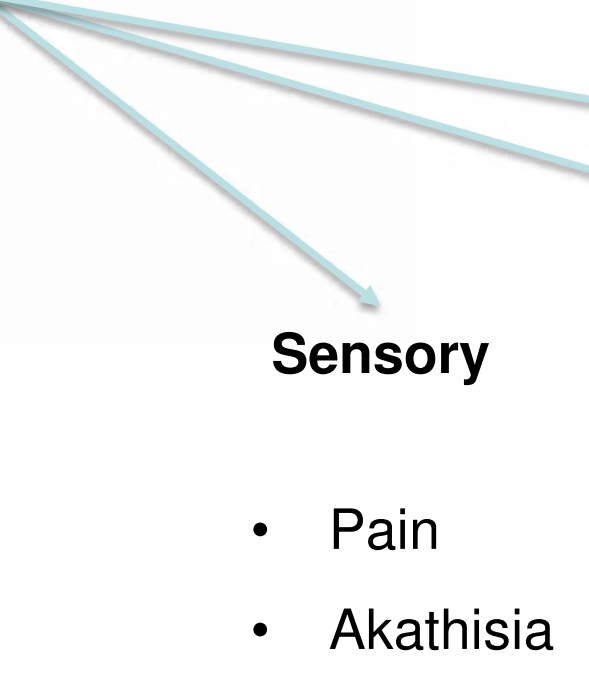

- Akathisia

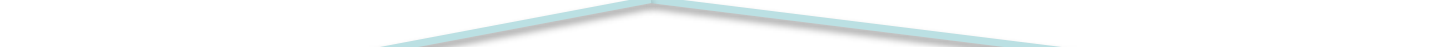

